\section{An Efficient Hybrid GO-PWS Algorithm to Analyze Conformal Serrated-Edge Reflectors for Millimeter-Wave Compact Range}

\author{
Alfonso Muñoz-Acevedo and Manuel Sierra-Castañer
}

\begin{abstract}
A method to analyze parabolic reflectors with arbitrary piecewise rim is presented in this communication. This kind of reflectors, when operating as collimators in compact range facilities, needs to be large in terms of wavelength. Their analysis is very inefficient, when it is carried out with fullwave/MoM techniques, and it is not very appropriate for designing with PO techniques. Also, fast GO formulations do not offer enough accuracy to reach performance results. The proposed algorithm is based on a GO-PWS hybrid scheme, using analytical as well as non-analytical formulations. On one side, an analytical treatment of the polygonal rim reflectors is carried out. On the other side, non-analytical calculi are based on efficient operations, such as $M^{2}$ order 2-dimensional FFT. A combination of these two techniques in the algorithm ensures real ad-hoc design capabilities, reached through analysis speedup. The purpose of the algorithm is to obtain an optimal conformal serrated-edge reflector design through the analysis of the field quality within the quiet zone that it is able to generate in its forward half space.
\end{abstract}

Index Terms-Compact range, hybrid algorithm, millimeter wave, plane wave spectrum, serrated edge reflector.

\section{INTRODUCTION}

Plane wave spectrum (PWS) theory offers a solution to Maxwell's equations representing the electromagnetic field distributions as a sum of independent plane waves. The simplicity, in terms of mathematical treatment, for each one of these elementary contributions, makes PWS formulations quite attractive when dealing with half-space radiation and propagation schemes in both near and far field conditions. Reflectors have classically been analyzed with different order physics techniques. The two most common techniques are GO and PO. As commented in [1], PO techniques are high frequency solving techniques, which offer a realistic solution to most problems. GO is a much simpler approach, while, as stated in [2], does not offer enough accuracy to carry out design—-stage tasks. Hybrid approaches to the problem combine different order electromagnetic physics in order to reach more accurate results and, if possible, computational speedup.

As studied in [3], PO techniques are in some cases insufficient to analyze serrated edge reflectors, since they assume a high-frequency hypothesis for which surface currents are not affected by edge effects at the serrated reflector rim. As pointed out in [3], [4], a common solution consists on using MoM techniques to evaluate the reflector's impressed currents and integrate them through PO techniques to calculate the radiated field. This approach is able to offer very accurate results of both near and far fields of the reflector, although it is highly inefficient for reflectors which are electrically very large, as those of CATRs are. Another hybrid approach to reach more realistic surface currents consists on assuming edge currents which also contribute to the radiated field (PTD techniques [2], [5]). Edge modeling is critical in the analysis and design of serrated-edge reflectors inside compact ranges. As commented in [6], simple approaches can offer sufficient results, while it is important to bound the maximum admissible error and also to be able to analyze different geometrical serrations alternatives [7]. GTD techniques [8] are an improvement of GO techniques, and are able to offer more accurate results by using information of the diffracted rays from the reflector's edges, but its utility is limited to scattering angles distant from the reflector's broadside, above a minimum reliable angle. GTD itself is not able to offer a complete solution to an arbitrary scattering problem. Common convergence criteria establish dense meshes $\left(\Delta x, y<\lambda_{o} / 30\right)$ over the surface currents [2]. Different techniques have been developed setting an adaptive mesh over the surface, or conformal meshing schemes [2], [9]. The evaluation of the Fresnel integral is itself a $\mathrm{O}\left(\mathrm{N}^{4}\right)$ complexity problem, given that for each one of the $O\left(\mathrm{~N}^{2}\right)$ points of interest, a $O\left(\mathrm{~N}^{2}\right)$ complexity integral must be performed, so PO techniques are a computational bottleneck when solving electrically large reflectors.

The motivation of performing integration through a plane wave spectrum (PWS) approach is the substantial reduction of the computational cost. PWS formulations evaluate the radiated fields of arbitrary scatterers through complex variable integral formulations and Fourier analysis [10]-[12]. In particular, parabolic scatterers are modeled with their aperture field distribution [13], in a half-space problem, which suits simple planar geometry formulations. The treatment of the planar field distribution is carried out through Fourier analysis, and practical implementations use of FFT. Thus, if the sampling of the field is carried out at general Nyquist rate $\left(\Delta x, y=\lambda_{o} / 2\right)$, the discretization of the aperture field is dense enough [11], [13]. These lower density meshes are an intrinsic computational advantage of plane wave spectrum formulations.

On the side of feed modeling, different order physics should be used depending on the particular setup to be analyzed. When low gain feeders and high $F / D$ facilities are used, as in the case of CATRs, the reflector's surface can be assumed to be in the far-field region of the feeder. Consequently, the impressed currents are calculated through GO techniques and feeder radiation pattern weighting. However, if this hypothesis is not accomplished, a complete near-field model of the feeder should be used to calculate the impressed currents distribution. Thus, PO techniques might be needed between the feeder and the reflector [15].

In the proposed algorithm, the impressed currents are projected to obtain the aperture distribution. As discussed in [15], this aperture field has an intrinsic phase error, due to the projection of the currents in the parabolic surface to the projected surface. The main consequence of computing the radiated field from this projected surface instead of the scatterer surface is the reduction of the angle validity in the radiated field. For the CATR case of study, scatterers with high $F / D$ figures do not introduce high phase errors, while CATRs operate in a narrow margin of radiation angles.

This communication is divided in five sections. After this introduction, Section II shows the mathematical formulation of the algorithm, explaining separately the PWS and the GO contributions. Section III offers field results of particular setups. Quantitative information is offered and field acquisitions are related to GRASP8 acquisitions. Section IV concludes some ideas for the use of this algorithm in the design of CATR facilities, and establishes the future lines. 


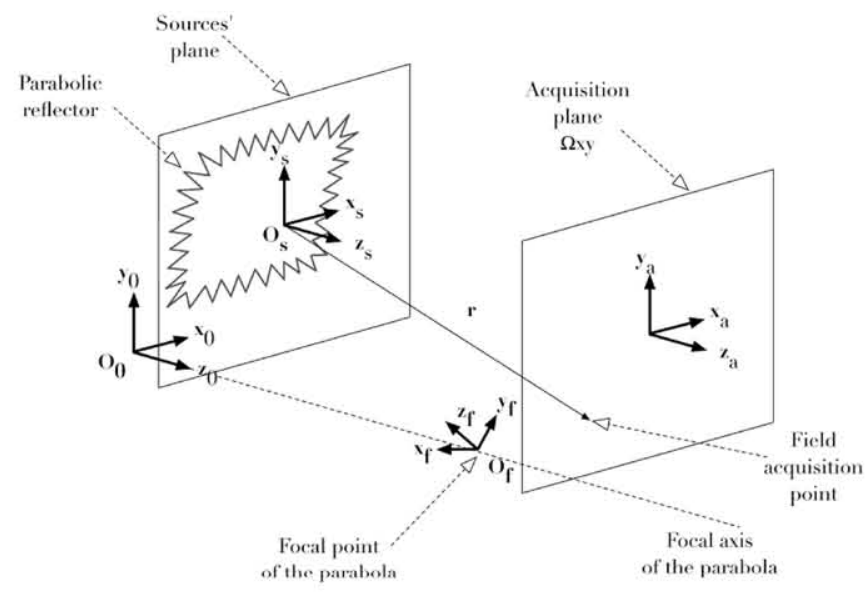

Fig. 1. Analysis problem: focused offset reflector CATR.

\section{MATHEMATICAL Formulation OF THE AlgORITHM}

\section{A. The Plane Wave Spectrum Representation}

As mentioned in the introduction, formulations of the Plane Wave Spectrum theory are shown for the planar acquisition surface canonical case. Clemmow studied deeply these formulations in [10]. There, it is shown that (1) and (2) constitute a solution to Maxwell's equations in a free space source-free region, where $\mathbf{r}$ is the acquisition point vector, respect to the sources distribution coordinate system $O_{s}$. This coordinate system is the one respect to which the plane wave spectrum of the fields is calculated

$$
\begin{aligned}
\mathbf{E}(\mathbf{r})= & \frac{1}{2 \pi} \int_{-\infty}^{\infty} \int_{-\infty}^{\infty} \tilde{\mathbf{E}}\left(k_{x}, k_{y}\right) e^{-j \mathbf{k} \cdot \mathbf{r}} d k_{x} d k_{y} \\
\mathbf{H}(\mathbf{r})= & \frac{1}{2 \pi} \cdot \frac{1}{Z_{0} \cdot k_{0}} \\
& \cdot \int_{-\infty}^{\infty} \int_{-\infty}^{\infty} \mathbf{k} \times \tilde{\mathbf{E}}\left(k_{x}, k_{y}\right) e^{-j \mathbf{k} \cdot \mathbf{r}} d k_{x} d k_{y} .
\end{aligned}
$$

Equations (1) and (2) become Fourier Transforms between the spatial $(x, y)$ domain and the spectral $\left(k_{x}, k_{y}\right)$ one. This approach requires the definition of the longitudinal propagation constant $k_{z}$, as in [12]. The transformed function of the field at the acquisition plane is the z-propagated plane wave spectrum distribution. The sources distribution is located at $z_{s}=0$ (Fig. 1), so the spectral distribution is obtained performing the inverse Fourier Transform shown in (3). A practical approach to this equation consists on setting a rectangular $x y$ mesh of $\mathrm{M}$ times $\mathrm{M}$ points over the $z_{s}=0$ plane, with uniform distance between samples $\Delta x=\Delta y=\lambda_{o} / 2$.

The continuous and vectorial $\mathbf{E}\left(x_{s}, y_{s}, z_{s}=0\right)$ field is sampled and separated in components, obtaining 3 scalar 2-dimensional discrete set of complex fields (for each one of the rectangular components $x, y$ and $z$ ). The Fourier transform of (3) becomes a DFT, which can be implemented with an efficient $M^{2}$ order 2-dimensional FFT

$\tilde{\mathbf{E}}\left(k_{x}, k_{y}\right)=\int_{-\infty}^{\infty} \int_{-\infty}^{\infty} \mathbf{E}\left(x_{s}, y_{s}, z_{s}=0\right) e^{j k_{x} \cdot x_{s}} \cdot e^{j k_{y} \cdot y_{s}} d x \cdot d y$.

\section{B. Sampling Criteria}

As pointed out in [13], Nyquist sampling in the spatial domain ( $\left.\Delta x=\Delta y=\lambda_{o} / 2\right)$ is enough to avoid the aliasing effects, in the most general case. Moreover, the extension of the spatial domain $\Omega_{\mathrm{xy}}$ must contain the region where the field is of interest. In particular $\Omega_{s} \subset \Omega_{\mathrm{xy}}$, where $\Omega_{s}$ is the minimum spatial support which includes the projected currents distribution, as defined in (4) and (5). If $\Omega_{\mathrm{xy}}$ is

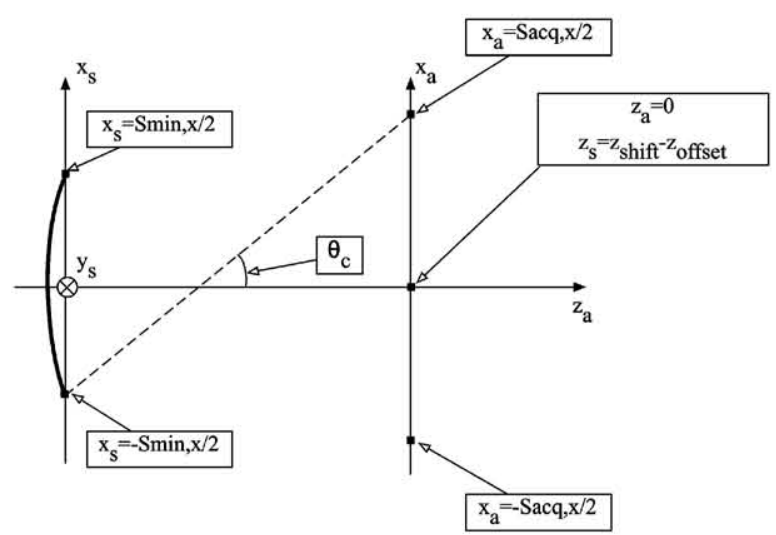

Fig. 2. Maximum usable angle seen by the reflector geometry.
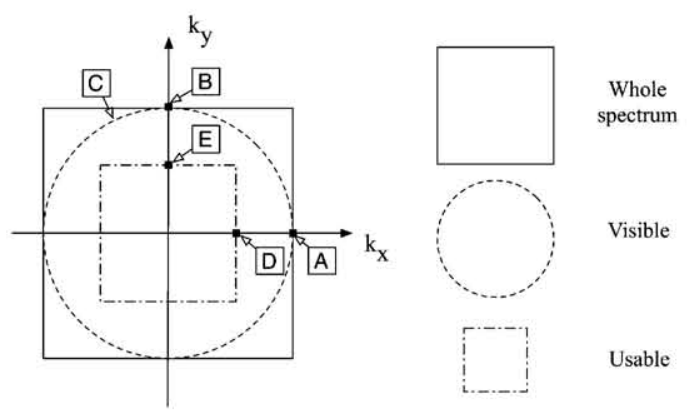

$$
\begin{aligned}
& \text { A. } k_{x}=k_{0} \\
& \text { B. } k_{y}=k_{0} \\
& \text { C. }\|k\|=k_{0} \\
& \text { D. } k_{x}=k_{x, \text { max }} \\
& \text { E. } k_{y}=k_{y, \max }
\end{aligned}
$$

Fig. 3. Spectral domains involved in the propagation problem.

larger than $\Omega_{s}$, there is a spatial "domain widening", which might be understood as an oversampling of the transformed variables $\left(k_{x} k_{y}\right)$ whenever the space between spatial samples is kept

$$
\begin{aligned}
\Omega_{S} & =\left[-\frac{S_{\min , x}}{2}, \frac{S_{\min , x}}{2}\right] \times\left[-\frac{S_{\min , y}}{2}, \frac{S_{\min , y}}{2}\right] \\
\Omega_{x y} & =\left[-\frac{S_{a c q, x}}{2}, \frac{S_{a c q, x}}{2}\right] \times\left[-\frac{S_{a c q, y}}{2}, \frac{S_{a c q, y}}{2}\right] .
\end{aligned}
$$

For this case, and without loss of generality, an oversampling vector $\eta$ can be defined as in (6)

$$
\begin{aligned}
\eta & =\left[\eta k_{x}, \eta k_{y}\right]=\left[\frac{S_{\min , x}}{2 \cdot S_{a c q, x}}, \frac{S_{\min , y}}{2 \cdot S_{a c q, y}}\right] \\
& =\left[\frac{M_{\min , x}}{2 \cdot M_{a c q, x}}, \frac{M_{\min , y}}{2 \cdot M_{a c q, y}}\right] .
\end{aligned}
$$

A $\boldsymbol{\eta}$ component close to 0 implies that the corresponding spectral variable is strongly oversampled, and the field is being calculated out of the reflector's projected surface for the transformed spatial direction. The modulus of the $2 \boldsymbol{\eta}$ vector states for the fraction of total field which is being calculated out of the reflector's projected surface. It is recommendable to choose components $\eta k_{x}, \eta k_{y} \leq 0.5$ that also make the number of samples per direction a power of two, in order to perform the calculations via FFT.

For a fixed $\Omega_{x y}$, the number of PWS modes that this domain is able to integrate is decreasing when increasing the $z_{s}$ distance between $\Omega_{s}$ and $\Omega_{\mathrm{xy}}$. This fact was already pointed out in [14], where some numerical examples for particular reflectors were given. It can be reached, moreover, an analytical expression for the required number of PWS modes to determine univocally the quiet zone field in $\Omega_{\mathrm{xy}}$. The explanation of this lies on the relationship between the spectral variables $\left(k_{x}, k_{y}\right)$ and their corresponding geometrical angles $\left(\theta_{x}, \theta_{y}\right)$, 

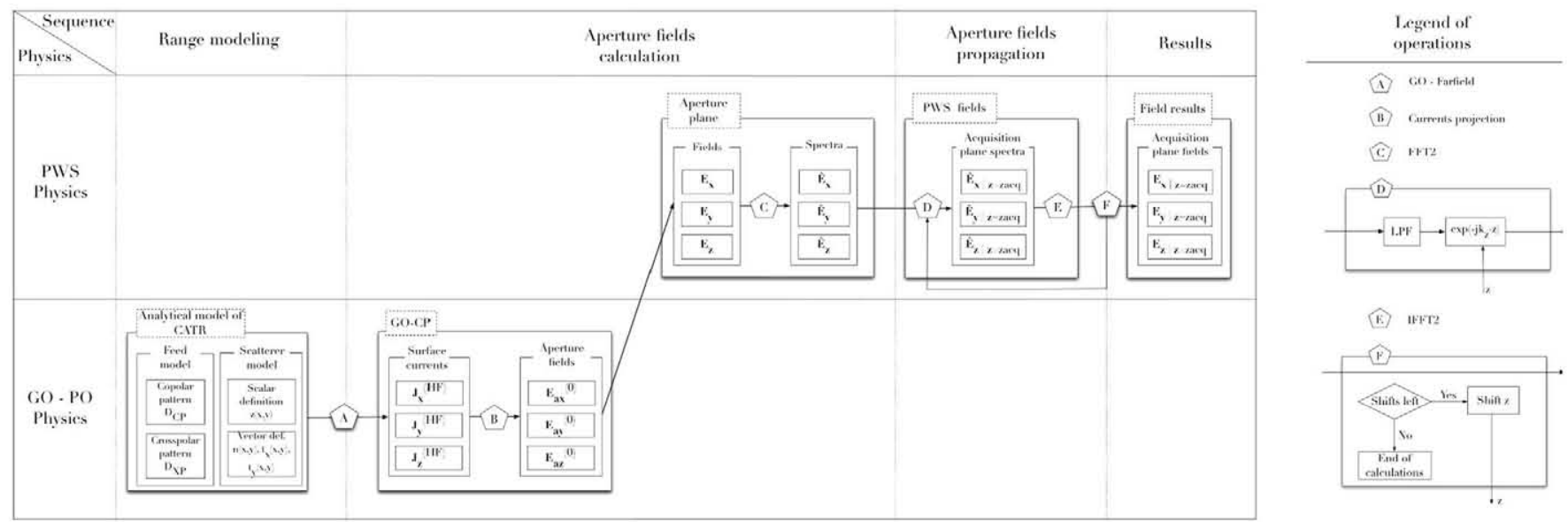

Fig. 4. Blocks scheme of the algorithm.

depending on the separation $\dot{\sim}_{s}$. between $\Omega_{s}$ and $\Omega_{\mathrm{xy}}$. It is easily concluded that $\left(k_{x} k_{y}\right)$ do not reach their boundary values $\pm k_{o}$ whenever $z_{s}>0$, and these bounds define the usable fraction of the whole complex spectrum (Fig. 2). Equations (7) and (8) relate $\left(k_{x} k_{y}\right)_{\max }$ with the geometrical and electromagnetic parameters. The spectral domain of these contributing modes is described with (9) and the amount of usable samples in a spatial direction is stated by (10)

$$
\begin{aligned}
{\left[k_{x}, k_{y}\right]_{\max } } & =k_{0} \cdot\left[\sin \left(\theta_{\max , x}\right), \sin \left(\theta_{\max , y}\right)\right] \\
\tan \left(\theta_{\max ,(x / y)}\right) & =\frac{S_{a c q,(x / y)}+S_{\min ,(x / y)}}{2 \cdot\left(z_{s}-z_{\text {off } f \text { set }}\right)} \\
& =\frac{M_{\min ,(x / y)} \cdot \lambda_{0}}{4 \cdot\left(z_{s}-z_{\text {off } f s t}\right)}\left(1+\frac{1}{2 \cdot \eta k_{(x / y)}}\right) \\
\tilde{\Omega}_{Q Z} & =\left[-k_{x, \max }, k_{x, \max }\right]\left[-k_{y, \max }, k_{y, \max }\right] \\
M_{u s a b l e(x / y)} & =\operatorname{int}\left(M_{w i d(x / y)} \cdot \sin \left(\theta_{\max ,(x / y)}\right)\right) .
\end{aligned}
$$

\section{GO Contributions to the Algorithm}

The setup to be analyzed, depicted in Fig. 1, consists of an offset parabolic shaped-edge reflector fed by a low gain horn, with an acquisition plane inside the Fresnel region of the reflector. There are three coordinate systems: $O_{0}$ is centered in the vertex of the parabolic surface and z-directed according to the propagation of the scattered field by the reflector. $O_{s}$ is centered in the projected surface of the reflector, with the same $z$ axis orientation as previous, but linearly shifted $z_{o f f s e t} . O_{f}$ is the feeder's local system, $\mathrm{z}$-directed with the maximum radiation pattern direction. The projected surface is located as close as possible to the reflector, but keeping all the surface currents at $z_{s}<0$ values.

A block scheme of the whole hybrid algorithm is shown in the matrix of Fig. 4. The lower row corresponds to the calculation of the aperture fields ( 2 nd column) through GO formulations (operation A) and current projection (op. C) over an analytical model of the CATR (1 st column). A legend of the operations involved in the task flow is attached. The aperture field distribution (2nd column) is calculated through GO techniques, since the use of GO is a simple approach to the field representation. Two main hypothesis are assumed to use GO in the feed modeling: first the reflector is in the farfield of the feeder; the second one requires a large reflector, in terms of wavelength [16], to assume the high frequency hypothesis for the surface currents. At low frequencies, for which the $\mathrm{GO}$ approach would be insufficient, a higher order formulation for the aperture fields should be added. In our case of study, the serrations are assumed to be large (much larger than 5 wavelengths), so HF assumption can be taken.

\section{Spectral Contributions to the Algorithm}

According to Fourier theory, the spatial window can be obtained by performing an IFT over the PWS of the projected surface. As presented in [12], [16]-[19], this calculation can be accurate and flexible for an arbitrary polygonal projected shape. In [14], it is approached through a convolution of the window's spectral distribution with the Fourier coefficients of the feeder's GO pattern projected over the acquisition plane, assuming that this pattern is spectrally very narrow banded. Our approach doesn't work on this assumption but avoids the spectra convolution by performing the windowing in the spatial domain. Moreover, the use of the closed form expression proposed in [17] avoids the truncation of rim information through analytical analysis of the geometry. Truncation effects can be noticed in [14], and can also be minimized if a conformal mesh of the serrations is considered, as proposed in [2].

The proposed approach requires two $M^{2}$-order $O\left(M^{2} \log M^{2}\right)$ FFT calculations (Operations C,E), in addition with a $O\left(M^{2}\right) M$ times $M$ multiplication (1st row, 1st column) to reach the spectral information of the reflector tapered by the feeder pattern. This spectral distribution is propagated towards the $z_{a}=0$ acquisition plane multiplying each discrete spectral plane wave contribution by a propagation factor (Op. D). The electric field at the acquisition plane $z_{a}=0$ is obtained performing a $M^{2}$-order FFT (Op. E) over the propagated spectrum. Operation $\mathrm{F}$ evaluates if more acquisition planes are of interest and, in that case, loops back to Op. D.

In practical terms, this means that the computation time used by the hybrid formulation is inelastic with respect to the number of acquisition planes and, thus, it is suitable for quiet zone volumetric characterization. It must be noted that the furthest the acquisition plane is set, the less time Ops. D, E take, since increasingly lower number of plane waves need to be processed. The tasks have been described for one field component, potentially the co-polar contribution. The process to obtain the other electric field components requires the analogous replication of columns 3 rd and 4 th for the other aperture field components.

\section{E. Accuracy of the Algorithm}

The hybrid algorithm is designed as a chain of different order Physics approximations based on certain hypothesis: the "feed in farfield" can be assumed for most CATR facilities and the "currents projection" approach should also be valid whenever the GO-PWS fields are of interest and the reflector is parabolic. However, the HF hypothesis is not valid if the frequency of operation is very low. Consequently, the accuracy analysis will focus on reaching an evaluator of the algorithm's reliability for this hypothesis. A pragmatic approach to this evaluation deals with the achievement of a signal to error figure which may determine 
if the proposed algorithm is valid to analyze a CATR reflector, for a particular setup.

It is realistic to obtain this signal to error figure through the energy ratio between two magnitudes (11). The numerator of this fraction is the best achievable approach to the quiet zone field (reference value). The denominator is the committed error when the quiet zone field is based on the HF approximation, as in (12).

$$
\begin{aligned}
\text { snr } & =\frac{\xi\left\{E_{Q Z}^{\aleph}(x, y ; z, \lambda)\right\}}{\xi\left\{E_{Q Z}^{*}(x, y ; z, \lambda)\right\}} \\
E_{Q Z}^{*}(x, y, z ; \lambda) & =E_{Q Z}^{\aleph}(x, y, z ; \lambda)-E_{Q Z}^{(G O-P W S)}(x, y, z ; \lambda) .
\end{aligned}
$$

It is convenient to express the numerator of (11) through Parseval's theorem (13). This expression states that only the PWS modes inside $\Omega_{\mathrm{QZ}}$ contribute to the energy $\xi$ of the reference distribution. It also states that the integration of the spectral distribution within $\Omega_{\mathrm{QZ}}$ at an arbitrary $z_{s}>0$ plane leads to the same $\xi$ figure as when the subintegral distribution is the spectrum existing at $z_{s}=0$.

$$
\begin{aligned}
\xi\{E(x, y ; z, \lambda)\} & =\iint_{\Omega x y} E(x, y ; z, \lambda) \cdot d x \cdot d y \\
& =\frac{1}{4 \cdot \pi^{2}} \iint_{\tilde{\Omega}_{Q Z}} \tilde{E}\left(x, y ; z_{s}=0, \lambda\right) d k_{x} \cdot d k_{y} .
\end{aligned}
$$

Some numeric results can be offered and particular signal to error figures can be extracted. To this purpose, the formulation used as reference is the time-domain full wave technique implemented by CST software. The test structure is a $400 \mathrm{~mm}$-wide PEC plate placed in $x y$ plane, over which a $y$-polarized plane wave impinges. Periodic boundary conditions on its $y$-axis and freespace boundary conditions over its $x$-axis are set. This offers a realistic approach for a 1-D analysis along the $x$ dimension. The purpose of this setup is to test the HF hypothesis for a fixed physical size scatterer between $5 \mathrm{GHz}$ and $80 \mathrm{GHz}$.

The induced homogeneous HF-currents $J_{\text {ind }}$ are compared with the full wave solution. These results are evaluated with (11) and (13) to reach the $s n r$ figures (Fig. 5(a)). The full-wave accuracy results are taken as the "reference" accuracy of the algorithm. It is relevant to point out the increase of accuracy obtained when moving up in frequency or shifting the acquisition plane away from the scatterer, as it is the algorithm's scenario of interest: mmWave CATRs. The scatterer's edge conformation is not considered with this canonical 1D problem. However, this lack of generality is overcome through the uniform illumination scheme, which is the HF hypothesis worst case scenario, given that it has the strongest impact on edge effects.

An empirical expression of SNR as a function of frequency is extracted from the full wave results. The logarithmic figure of accuracy $S N R(f[G H z])$ is best-fit to a second order polynomial, as in (14), and the $a_{i}$ coefficients (Fig. 5(b)) are calculated along the evaluated longitudinal shift domain. For sake of generality, it is also reached (15), which draws the SNR figure from the electrical size of the scatterer $\Lambda$, in wavelengths. This is obtained bearing in mind that the physical size of the scatterer remains constant and equal to $400 \mathrm{~mm}$ among all the simulations

$$
\begin{aligned}
S N R_{d B}\left(f_{G H z}\right) & =\sum_{i=0}^{2} a_{i} \cdot\left(f_{G H z}\right)^{i} \\
S N R_{d B}(\Lambda) & =\sum_{i=0}^{2} a_{i} \cdot\left(\frac{3}{4} \cdot \Lambda\right)^{i} .
\end{aligned}
$$

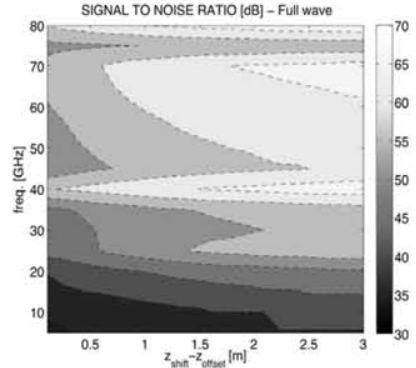

(a)

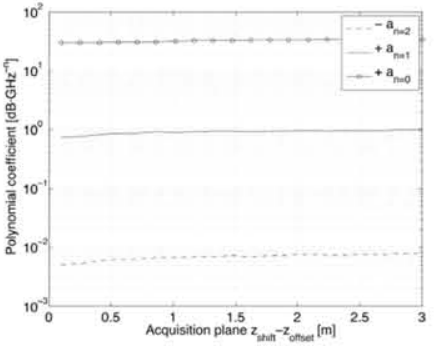

(b)
Fig. 5. (a) Logarithmic SNR vs. frequency and acquisition plane distance using as reference the CST calculation and (b) the polynomial best fit SNR figure, as a function of the acquisition plane distance.
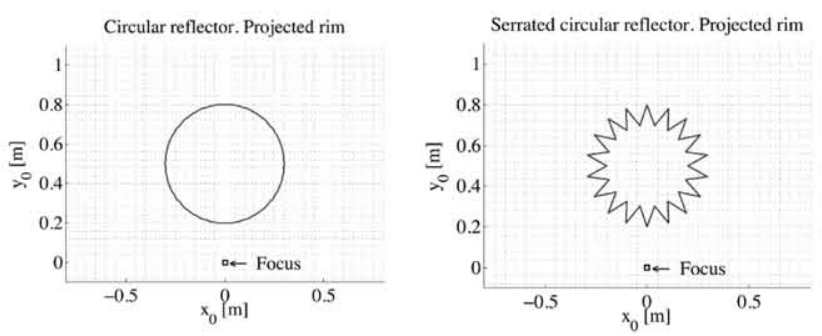

Fig. 6. Circular and serrated reflector projected rim respect to $O_{o}$ coord. sys.
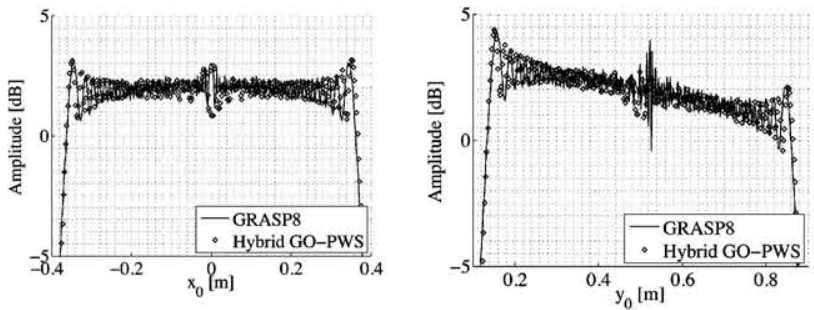

Fig. 7. Normalized amplitude, horizontal and vertical cuts.

\section{RESULTS-VALIDATION}

Our purpose is to obtain plane acquisitions of electric field inside the Fresnel region of a serrated-edge reflector. Thus, the results to be studied will be E/H-plane cuts of the diffracted electric field, in a compact-range, where the feeder is pointing to the center of the reflector. The obtained results are compared to electric field results from GRASP8, that is the reflector analysis tool more widely used to carry out PO analysis over reflector antennas [22].

\section{A. Circular Offset Reflector}

A first test structure is a circular offset scatterer, with the acquisition setup proposed in Fig. 1. The corresponding parameters of simulation are indicated in Table I. The circular projected surface is modeled by constructing a 60 vertices piecewise polygonal rim. Isotropic feeder is chosen in order to weight as uniformly as possible all the surface contributions on the scatterer and, thus, avoid weighting down certain regions, which could mask algorithm eventual inaccuracies. Normalized amplitude and phase for both $\mathbf{E}$ and $\mathbf{H}$ planes are depicted in Figs. 7 and 8. As it can be deduced from these figures, good agreement is achieved, in general terms, between PO and GO-PWS results. 

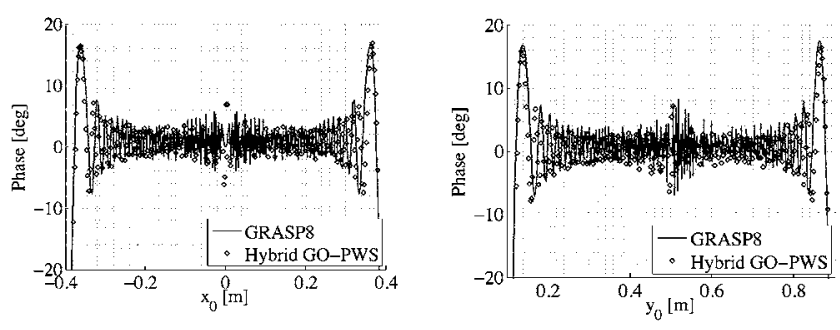

Fig. 8. Normalized phase, horizontal and vertical cuts.

TABLE I

THST RhH.hCTOR A: CiRCUI.AR RhH.FCTOR SimUi.ATION

\begin{tabular}{ll}
\hline \multicolumn{1}{c}{ Parameter } & Value \\
\hline Frequency & $300 \mathrm{GHz}$ \\
$\mathrm{D}$ & $0.6 \mathrm{~m}$ \\
Doffset & $0.2 \mathrm{~m}$ \\
focal distance & $0.8 \mathrm{~m}$ \\
zshift & $1.0 \mathrm{~m}$ \\
zoffset & $0.2 \mathrm{~m}$ \\
Feed pattern & Isotropic \\
\hline
\end{tabular}

TABLE II

Thist Rhilitctor B: Serrathid Circular Rhilifctor Simulation

\begin{tabular}{ll}
\hline \multicolumn{1}{c}{ Parameter } & \multicolumn{1}{c}{ Value } \\
\hline Frequency & $100 \mathrm{GHz}$ \\
$\mathrm{D}$ & $0.6 \mathrm{~m}$ \\
& $\quad$ Internal radius: $0.2 \mathrm{~m}$ \\
& Serration depth: $0.1 \mathrm{~m}$ \\
Doffset & $0.2 \mathrm{~m}$ \\
focal distance & $2.5 \mathrm{~m}$ \\
zshift & $3.0 \mathrm{~m}$ \\
zoffset & $0.128 \mathrm{~m}$ \\
Feed pattern & Gaussian: $-1.0 \mathrm{~dB}$ for $\theta=10^{\circ}$. \\
& Independent with $\phi$. \\
\hline
\end{tabular}
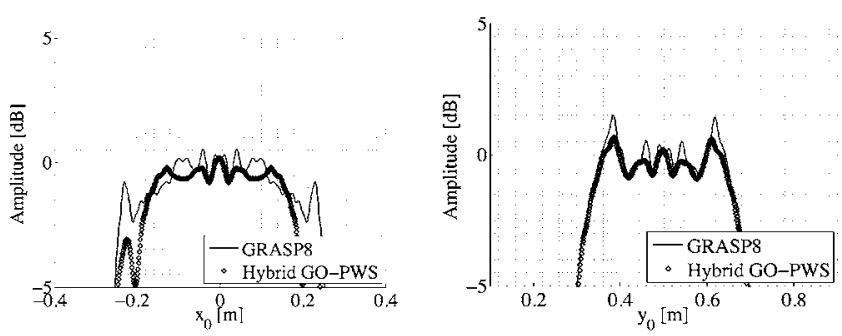

Fig. 9. Normalized amplitude, horizontal and vertical cuts; serrated reflector.

\section{B. Serrated Circular Offset Reflector}

A circular offset reflector with linear serrated edge is now studied. The setup is the same as previous, with the definition of Table II. This second reflector has a higher $\mathrm{F} / \mathrm{D}$, which minimizes the amplitude slope in the vertical cut; 36 test serrations of $0.1 \mathrm{~m}$ length are placed around a circular rim with $0.2 \mathrm{~m}$ of radius.

The feeder follows a Gaussian pattern, with $-1 \mathrm{~dB}$ at $\theta=10^{\circ}$ and non $\phi$-dependent. Amplitude and phase cuts are shown in Figs. 9 and 10. A good agreement is observed for both magnitudes in the vertical cut, whereas horizontal acquisition has not the same level of agree-
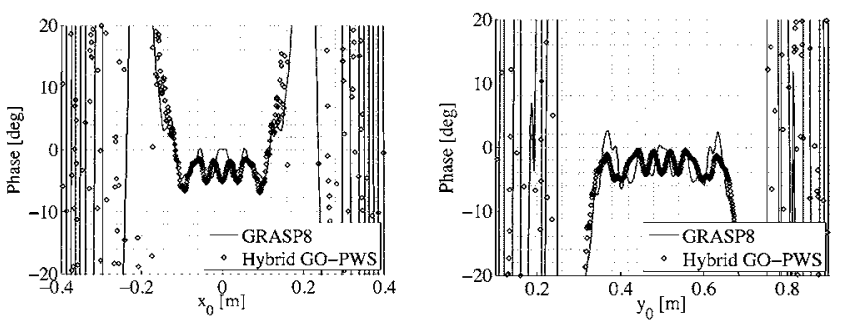

Fig. 10. Normalized phase, horizontal and vertical cuts; serrated reflector.

ment. The analysis performed in [16] using MoM, observes similar discrepancies between PO and MoM. In any case, this hybrid method can give accurate results to bound the ripple and the tapering in a single reflector CATR, if the hypotheses introduced in Section II-C are accomplished.

\section{Performance of the Algorithm}

After simulation results have been shown, speedup notes must be added to offer a clear idea of the algorithm's global performance. Simulation times are considered, as overall indicators. The algorithm has been implemented in FORTRAN code, and uses high performance FFT external modules. Simulations were carried out in a $2.8 \mathrm{GHz}$ double core Pentium 4 processor, with 4 GB of RAM. GRASP8 took $51.200 \mathrm{~s}$ and $31.500 \mathrm{~s}$ to reach the results concerning reflectors $\mathrm{A}$ and $\mathrm{B}$ respectively. On the other side, the implementation of the algorithm took 47 $\mathrm{s}$ and $11 \mathrm{~s}$ to reach its results.

\section{CONCLUSIONS: APPLiCATION TO CATR DESIGN}

The development of this algorithm has, as main goal, to speed up the design of conformal serrated edge reflectors operating as wave collimators in compact range facilities. By performing serrations in the edge of parabolic reflectors, amplitude as well as phase ripples of the diffracted electric field decrease. Thus, the field distribution gets closer to the local plane one, which is our ideal situation. Typical peak to peak values are $1 \mathrm{~dB}$ for amplitude and 10 degrees for phase. The design process uses as unknowns the geometrical variables which define the CATR facility, while the cost functions are the amplitude and phase ripple of the electric field inside the quiet zone. High time-consuming unitary simulations have as main consequence prohibitive or, at least, incomplete design processes. The use of efficient algorithms, such as the one proposed in this communication, is necessary if complete design processes are desired.

In this work, an algorithm to analyze arbitrary piecewise rim reflectors has been presented. The propagation problem was divided into regions, for each one of which, a different analysis solution was used. To carry out joint optimization of the different order electromagnetic physics, a deep study of the field sampling was presented and analytical formulation of the sampling problem was proposed for the general case. These formulations were implemented in order to reach both more accurate results and a computational speedup. Some clear ideas were given about the extension of the algorithm to higher order physics.

In order to test the algorithm, a closed form expression of the algorithm's accuracy was reached and experimentally developed with a full wave method. Moreover, field acquisitions were compared with theoretical results obtained through GRASP8, which uses PO-order techniques, for two different canonical reflectors.

\section{REFERENCES}

[1] H. F. Schluper, "Compact antenna test range analysis using physical optics," in Proc. 1987 Antenna Measurement Techniques Association Symp., Seattle, WA, pp. 309-312. 
[2] Y.-C. Chang and J. I. M. Jim, "A PTD analysis of serrated edge compact range reflectors," in Proc. 1994 Antenna Measurement Techniques Association Symp., Long Beach, CA, pp. 175-179.

[3] F. Jensen, L. Giauffret, and J. Marti-Canales, "Modeling of compact range quiet-zone fields by PO and GTD," in Proc. 1999 Antenna Measurement Techniques Association Symp., Monterey Bay, CA, pp. 242-247.

[4] A. Miura and Y. Rahmat-Samii, "Spaceborne mesh reflector antennas with complex weaves: Extended PO/Periodic-MoM analysis," IEEE Trans. Antennas Propag., vol. 55, no. 4, Apr. 2007.

[5] A. Michaeli, "Elimination of infinities in equivalent edge currents, part I: Fringe current components," IEEE Trans. Antennas Propag., vol. 34, no. 7, Jul. 1986.

[6] F. Jensen and K. Pontoppidan, "Modeling of the antenna-to-range coupling for a compact range," in Proc. 2001 Antenna Measurement Techniques Association Symp., Denver, CO, pp. 387-391.

[7] S. C. van Someren Greve, L. G. T. van de Coevering, and V. J. Vokurka, "On design aspects of compact antenna test ranges for operation below 1 GHz," in Proc. 2001 Antenna Measurement Techniques Association Symp., Monterey Bay, CA, pp. 248-253.

[8] M. Ando, "PO and PTD analyses of offset reflector antenna patterns," presented at the Antennas and Propagation Society Int. Symp., 1988.

[9] S. Kulkarni, R. Lemdiasov, R. Ludwig, and S. Makarov, "Comparison of two sets of low-order basis functions for tetrahedral VIE modelling," IEEE Trans. Antennas Propag., vol. 52, no. 10, Oct. 2004.

[10] P. A. Clemmow, The Plane Wave Spectrum Representation of Electromagnetic Fields, Reissued ed. Piscataway, NJ: IEEE Press, 1996

[11] R. J. Pogorzelski, "Improved efficient field computation via fast Fourier transforms," IEEE Antennas Wireless Propag. Lett., vol. 4, pp. 27-30, 2005.

[12] S. Liao and R. J. Vernon, "A fast algorithm for computation of electromagnetic wave propagation in half-space," IEEE Trans. Antennas Propag., vol. 57, no. 7, pp. 2068-2075, Jul. 2009.

[13] J. J. H. Wang, "An examination of the theory and practices of planar near-field measurement," IEEE Trans. Antennas Propag., vol. 36, no. 6, pp. 746-753, Jun. 1988.

[14] J. P. McKay and Y. Rahmat-Samii, "Compact range reflector analysis using the plane wave spectrum approach with an adjustable sampling rate," IEEE Trans. Antennas Propag., vol. 39, no. 6, pp. 746-753, Jun. 1991.

[15] Hu, M. Arrebola, R. Cahill, J. A. Encinar, V. Fusco, H. S. Gamble, Alvarez, Yu, and F. Las-Heras, "94 GHz dual-reflector antenna with reflectarray subreflector," IEEE Trans. Antennas Propag., vol. 57, no. 10, pp. 3043-3050, Oct. 2009.

[16] F. Jensen, "Polarization dependent scattering from the serrations of compact ranges," in Proc. AMTA 2007, St. Louis, MO, pp. 150-155.

[17] S.-W. Lee and R. Mittra, "Fourier transform of a polygonal shape function and its application in electromagnetics," IEEE Trans. Antennas Propag., vol. 31, no. 1, pp. 99-103, Jan. 1983.

[18] T.-H. Lee and W. D. Burnside, "Performance trade-off between serrated edge and blended rolled edge compact range reflectors," IEEE Trans. Antennas Propag., vol. 44, no. 1, pp. 87-96, Jan. 1996.

[19] P. A. Beeckman, "Prediction of the Fresnel region field of a compact antenna test range with serrated edges," IEE Proc. Microw. Antennas Propag., vol. 133, no. 2, pp. 108-114, Apr. 1986.

[20] P. A. Beeckman, "Control of far-field radiation patterns of microwave reflector antennas by using serrated edges," IEE Proc. Microw. Antennas Propag., vol. 134, no. 3, pp. 270-274, Jun. 1987.

[21] G. Parini and M. Philippakis, "Use of quiet zone prediction in the design of compact antenna test ranges," IEEE Trans. Antennas Propag., vol. 143, no. 3, pp. 193-199, Jun. 1996.

[22] R. L. Lewis and A. C. Newell, "An efficient and accurate method for calculating and representing power density in the near-zone of microwave antennas," IEEE Trans. Antennas Propag., vol. AP-36, no. 6, Jun. 1988

[23] E. B. Joy and R. E. Wilson, "Shaped edge serrations for improved compact range performance," in Proc. Antenna Measurement Techniques Association Meeting, Ottawa, Sep. 1986, pp. 23-25.

[24] A. Muñoz-Acevedo, M. Sierra-Castañer, and J. L. Besada, "Efficient and accurate hybrid GO-spectral algorithm to design conformal serrated-edge reflectors operating as collimators in millimeter wave compact ranges," presented at the 2010 Proc. Antenna Measurement Techniques Association Symp., Atlanta.

\section{Time-Domain Microwave Imaging of Inhomogeneous Debye Dispersive Scatterers}

\author{
Theseus G. Papadopoulos and Ioannis T. Rekanos
}

Abstract-A time-domain inverse seattering method for the reconstruction of inhomogeneous dispersive media described by the Debye model is presented. The method aims to the simultaneous reconstruction of the spatial distributions of the optical and static permittivity as well as of the relaxation time. The reconstruction of the scatterer is based on the minimization of a cost functional, which describes the difference between measured and estimated values of the electric field. The fulfillment of the Maxwell's curl equations is set as constraint by means of Lagrange multipliers in an augmented functional. The Fréchet derivatives with respect to the scatterer properties are derived analytically and can be utilized by any gradientbased optimization technique. The proposed reconstruction technique is based on the Polak-Ribière nonlinear conjugate-gradient algorithm, while the finite-difference time-domain (FDTD) method is employed for the solution of the direct and the adjoint electromagnetic problem. Numerical results for the reconstruction of one-dimensional layered scatterers illustrate the performance of the proposed method.

Index Terms-Debye model, dispersive media, finite-difference time-domain (FDTD), inverse scattering, Lagrange multipliers.

\section{INTRODUCTION}

Microwave imaging is an electromagnetic inverse scattering problem of significant interest because of its numerous applications in medical imaging, geophysical prospecting, nondestructive testing, etc. In general, the problem can be described as follows. Given a set of known electromagnetic wave excitations that illuminate a scatterer, the total or the scattered field is measured at locations around the scatterer domain. The objective is to reconstruct the spatial distribution of the electromagnetic properties of the scatterer by inverting the measurement data. This problem is nonlinear because the scattered field is a nonlinear function of the scatterer properties. Furthermore, it is an ill-posed problem because the operator that maps the scatterer properties to the scattered field is compact [1].

Depending on the time variation of the excitations used, two approaches and associated methodologies appear. In the first approach, namely the frequency-domain one, the excitation is considered monochromatic and the inverse scattering problem is formulated in the frequency domain by neglecting the time dependence of the fields [2]-[7]. In single frequency-domain microwave imaging, the case of dispersive scatterer properties is treated similarly to the case of nondispersive ones, because dispersion phenomena are not generated by monochromatic excitations. Finally, the reconstruction resolution of the scatterer properties is governed and actually limited by the wavelength of the excitation [5], [8]. It should be mentioned that frequency-domain microwave imaging could still be applicable to the case of dispersive scatterers, if multiple distinct excitation frequencies are utilized. To improve the reconstruction resolution and at the same time to cope with dispersive scatterers, a second microwave imaging approach, namely the time-domain one, has been proposed where the excitation field is wideband and the microwave imaging problem is

Manuscript received April 19, 2011; revised June 30, 2011; accepted August 03, 2011. Date of publication October 21, 2011 ; date of current version February $03,2012$.

The authors are with the Physics Division, School of Engineering, Aristotle University of Thessaloniki, GR-54124 Thessaloniki, Greece (e-mail: thiseasp@gen.auth.gr; rekanos@auth.gr). 